\title{
Characterization of BNC Type Calibration Standards
}

\author{
Murat Celep*, Erkan Danac1, and Handan Sakarya \\ TÜBİTAK Ulusal Metroloji Enstitüsü, Dr. Zeki Acar Cad. No:1, Gebze-Kocaeli, Turkey
}

\begin{abstract}
In recent years, BNC type devices such as attenuator and termination are used for high frequency measurements. Unfortunately, there is no any primary standard to obtain traceability for BNC type devices. It is proposed type $\mathrm{N}$ to $\mathrm{BNC}$ adapter characterization approach to obtain traceability for BNC type calibration standards, opens, shorts and matched loads used for calibration of BNC type devices. Electrical delay is calculated to characterize adapter then it is used for measurement of BNC type calibration standards. To validate proposed approach, known type $\mathrm{N}$ standards are measured and compared with actual results. Obtained results show good agreement. Difference between measured and actual phase for type $\mathrm{N}$ opens and shorts is lower than $8^{\circ}$ up to $10 \mathrm{GHz}$.
\end{abstract}

\section{Introduction}

The BNC (Bayonet Neill Concelman) connectors are used for coaxial cables since 1951. BNC is originally designed for military use and widely used in radio, television, radio frequency electronic equipment, test instruments and video signal applications and preferred due to easy connect/disconnect feature [1]. In general, maximum working frequency of BNC connectors is 3 $\mathrm{GHz}$ used for industrial applications. However, there are some devices and accessories with BNC connectors working more than $3 \mathrm{GHz}$ in the market in recent years $[2,3]$. Some of BNC connecterized devices are used for metrological manner such as attenuator and termination. Also, there are different type of BNC calibration kits working up to $4 \mathrm{GHz}$ and $10 \mathrm{GHz}[4,5]$.

Reference plane is one of parameters to define connectors. It must be defined for each connector family. Although, definition of the metrological connector's reference plane can be found at IEEE 287 standard [6], there is no any information about reference plane for BNC connectors. Also, there are no traceable primary standards for equipment with $\mathrm{BNC}$ connectors but $\mathrm{BNC}$ connecterized devices must be calibrated through traceable standards for metrological applications. In this study, characterization of adapter through traceable standards to calibrate BNC loads such as opens, shorts and matched loads is described.

\section{Characterization of standards}

\subsection{Type $\mathrm{N}$ to BNC adapter characterization}

A type $\mathrm{N}$ to $\mathrm{BNC}$ adapter which is traceable to international reference standard is used to characterize BNC standards. To get traceability, $\mathrm{N}(\mathrm{m})$ to BNC (m) and BNC (f) to $\mathrm{N}$ (f) adapters (Maury microwave
8821D1 and 8821A1 model) are connected together and obtained $\mathrm{N}(\mathrm{m})$ to $\mathrm{N}(\mathrm{f})$ adapter pair. There are two parameters of the adapter pair. One of them is transmission magnitude and the other one is transmission phase. These parameters are measured with R\&S ZVA50 vector network analyzer (VNA). First of all, type $N$ phase stable VNA cables which are suitable with adapter pair connectors connected to the VNA and frequency range of the VNA has been set from $50 \mathrm{MHz}$ to $10 \mathrm{GHz}$ with $10 \mathrm{MHz}$ step. Also, VNA IF bandwidth is settled to $10 \mathrm{~Hz}$ to obtain better result and $10 \mathrm{MHz}$ external reference is applied to the VNA to avoid frequency shift. Then, measurement system is calibrated using short, open, matched load and through calibration technique using suitable type $\mathrm{N}$ calibration kit (Agilent 85052B). Aforementioned adapter pair measurement setup and calibrated reference plane are shown in Figure 1.a. Transmission signal magnitude and phase parameters through the adapter pair inserted between the reference plane 1 and 2 are measured using setup Figure 1.a. Measured phase parameter is given in Figure 1.b.

There are four phase changing points when analyze Figure 1.b. With an additional VNA calibration and adapter pair measurement, these frequency points are defined to produce exactly same phase for each point. Frequency difference between two sequential points is related to electrical length of the adapter pair. So, electrical length of the adapter pair can be calculated through calculated frequency difference. Three frequency differences are calculated from four phase changing points. Electrical delay is calculated for each frequency difference point. Calculated frequency differences and electrical delays are given in Table 1. To investigate repeatability, reproducibility and dielectric effect of the connector, eight different measurements are performed with different connector orientations at whole frequency range.

\footnotetext{
Corresponding author: murat.celep@tubitak.gov.tr
}

(C) The Authors, published by EDP Sciences. This is an open access article distributed under the terms of the Creative Commons Attribution 


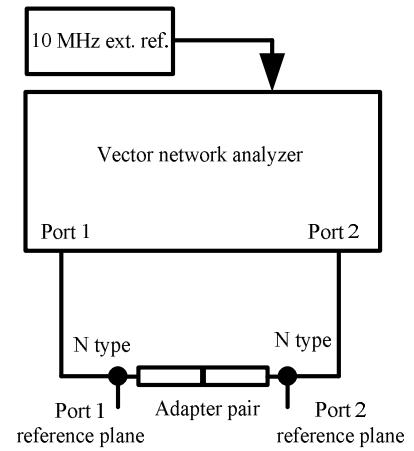

Fig. 1.a. Measurement setup of $N(m)$ to $B N C(m)$ and $B N C$ (f) to $\mathrm{N}(\mathrm{f})$ adapter pair.

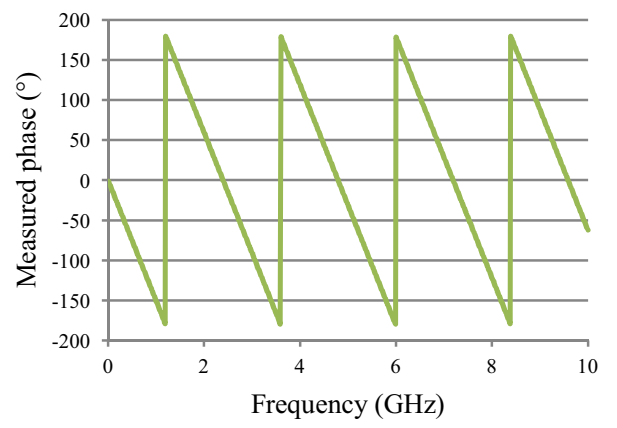

Fig. 1.b. Measured phase of $\mathrm{N}(\mathrm{m})$ to $\mathrm{BNC}(\mathrm{m})$ and $\mathrm{BNC}(\mathrm{f})$ to $\mathrm{N}$ (f) adapter pair up to $10 \mathrm{GHz}$.

Average and uncertainty which includes connector repeatability, reproducibility, dielectric effect, frequency drift and resolution are calculated from all delays as $417.36 \mathrm{ps}$ and $\pm 0.11 \mathrm{ps}$ respectively for adapter pair given Figure 2. Then mechanical length of each adapter is measured from reference plane to reference plane. Reference plane for BNC side of the adapter is chosen similar to type $\mathrm{N}$ connector. Ratio of mechanical measurements of the adapters is determined to minimize effect coming from difference between mechanical and electrical length. Finally, electrical delay for each adapter is calculated from adapter pair's delay and mechanical measurement's ratio. Same measurement method is also applied to $\mathrm{N}(\mathrm{m})$ to $\mathrm{BNC}(\mathrm{f})$ and $\mathrm{BNC}(\mathrm{m})$ to $\mathrm{N}$ (f) adapters (Maury microwave $8821 \mathrm{C} 1$ and 8821B1 model) to calculate delay. Calculated delay and uncertainty for each adapter and manufacturer specifications are given in Table 2. Attenuation of the adapter pairs are also measured with the VNA. It is assumed that each adapter part of adapter pair has same attenuation. So, the measured attenuation $(\mathrm{dB})$ is divided to two to find attenuation for each adapter.

Table 1. Calculated frequency differences and electrical delays for one orientation at whole frequency range for Maury microwave 8821A1 and 8821D1 model adapter pair.

\begin{tabular}{|c|c|}
\hline $\begin{array}{c}\text { Frequency } \\
\text { difference } \\
(\mathrm{GHz})\end{array}$ & $\begin{array}{c}\text { Delay } \\
(\mathrm{ps})\end{array}$ \\
\hline 2.396065107 & 417.35 \\
\hline 2.395901090 & 417.38 \\
\hline 2.396854185 & 417.21 \\
\hline
\end{tabular}

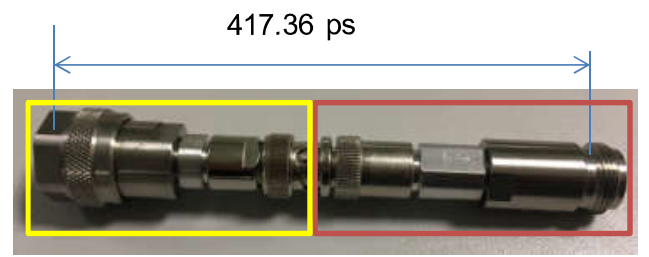

Fig. 2. Measured N to BNC adapter pair.

Table 2. Calculated delays and manufacturer specifications for Maury microwave $\mathrm{N}$ to BNC adapters.

\begin{tabular}{|c|c|c|}
\hline Model & Delay & $\begin{array}{c}\text { Manufacturer } \\
\text { specification }\end{array}$ \\
\hline $8821 \mathrm{~A} 1$ & $\begin{array}{c}194.34 \mathrm{ps} \\
\pm 0.62 \mathrm{ps}(\mathrm{k}=2)\end{array}$ & $195 \mathrm{ps}$ \\
\hline $8821 \mathrm{~B} 1$ & $\begin{array}{c}194.25 \mathrm{ps} \\
\pm 0.87 \mathrm{ps}(\mathrm{k}=2)\end{array}$ & $193 \mathrm{ps}$ \\
\hline $8821 \mathrm{C} 1$ & $\begin{array}{c}222.94 \mathrm{ps} \\
\pm 0.87 \mathrm{ps} \mathrm{(k=2)}\end{array}$ & $226 \mathrm{ps}$ \\
\hline $8821 \mathrm{D} 1$ & $\begin{array}{c}223.02 \mathrm{ps} \\
\pm 0.62 \mathrm{ps} \mathrm{(k=2)}\end{array}$ & $223 \mathrm{ps}$ \\
\hline
\end{tabular}

\subsection{BNC standards characterization}

Maury microwave $8550 \mathrm{G}$ model calibration kit which is specified up to $10 \mathrm{GHz}$ including opens, shorts and matched loads with BNC-male and -female connectors is used as BNC standards. The type $\mathrm{N}$ to $\mathrm{BNC}$ adapters with calculated electrical delay and attenuation are used to characterize BNC standards. Measurement setup and model to measure opens, shorts and matched loads are given in Figure 3. The type $\mathrm{N}$ to $\mathrm{BNC}$ adapter is connected to the VNA reference plane to measure reflection coefficient of open, short and matched load connected to BNC port of the adapter.

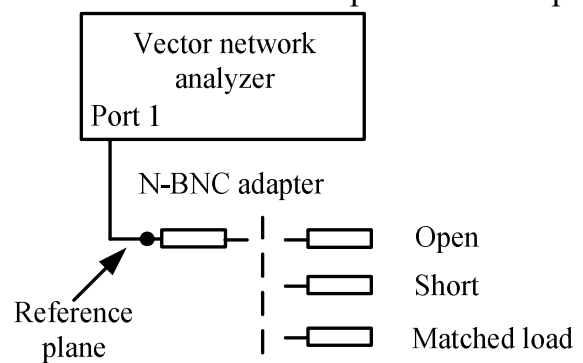

Fig. 3.a. Measurements setup

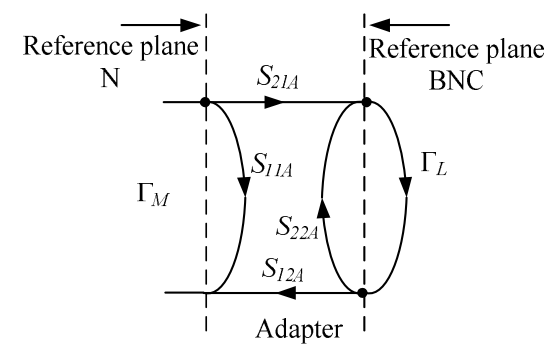

b)

Fig. 3.b. model for BNC calibration standards.

Mathematical model of measurement setup given in Figure 3.b is known very well and its equation is given as, 


$$
\Gamma_{M}=S_{11 A}+\frac{S_{12 A} \cdot S_{21 A} \cdot \Gamma_{L}}{1-S_{22 A} \cdot \Gamma_{L}}
$$

Where, $\Gamma_{M}$ is measured equivalent reflection coefficient of the adapter and load (open, short or matched load) combination. $S_{11 A}, S_{12 A}, S_{21 A}$ and $S_{22 A}$ are S-parameters of the adapter and $\Gamma_{L}$ is reflection coefficient of the measured load.

Reference plane in Figure 3.a is defined through type $\mathrm{N}$ calibration standards. If adapter's S-parameters are known, reflection coefficient of load can be calculated. Transmission coefficients $\left(S_{12 A}=S_{21 A}\right)$ of the $\mathrm{N}$ to BNC adapter are already calculated but reflection coefficients are unknown. It is assumed that $S_{11 A}$ and $S_{22 A}$ are equal to zero for simplification $\left(S_{11 A}=S_{22 A}=0\right)$. Phase of male open (open (m)), female open (open (f)), male short (short (m)) and female short (short (f)) are calculated using adapter transmission coefficient $\left(S_{I 2 A}\right.$ and $\left.S_{2 I A}\right)$ and measured reflection coefficient $\left(\Gamma_{M}\right)$. Calculated phases and manufacturer phase specifications of $\mathrm{BNC}$ opens and shorts for male- and female-connector are given in Figure 4.a and Figure 4.b respectively. Maximum phase uncertainty for measurements is $4.5^{\circ}$. Phase difference between measured and specification are given in Figure 5. Sharp peak and deep points are caused by phase change from $-180^{\circ}$ to $+180^{\circ}$. Obtained maximum differences are $16^{\circ}, 6^{\circ}, 19^{\circ}, 7^{\circ}$ for open (m), open (f), short $(\mathrm{m})$ and short (f) respectively. To calculate reflection coefficient of matched load, adapter reflection coefficient of each adapter's port is calculated by assuming that magnitude of reflection coefficient is 1 and then it is calculated using Equation 2.

$$
\Gamma_{L}=\frac{1}{S_{22 A}+\frac{S_{21 A} \cdot S_{12 A}}{\Gamma_{M}-S_{11 A}}}
$$

Calculated reflection coefficients of male and female matched loads are shown in Figure 6.

\section{Method validation}

To validate measurement and calculation method, type $\mathrm{N}$ calibration standards (Agilent $85054 \mathrm{D}$ calibration kit) are used as a characterized device. Type N (f) to type $\mathrm{N}$ (f) and type $\mathrm{N}(\mathrm{m})$ to type $\mathrm{N}(\mathrm{m})$ adapters, opens, shorts and matched loads with male and female connectors are used for validation. Type N (f) to type N (f) adapter is connected to type $\mathrm{N}(\mathrm{m})$ to type $\mathrm{N}(\mathrm{m})$ adapter to obtain adapter pair. The completely same described method is applied to measure adapter pair and delay is calculated as $330.65 \pm 0.11$ ps. Manufacturer specification for the adapter pair is given as 330.82 ps. Calculated delay for type $\mathrm{N}(\mathrm{f})$ to type $\mathrm{N}(\mathrm{f})$ and $\mathrm{N}(\mathrm{m})$ to type $\mathrm{N}(\mathrm{m})$ adapters are $196.85 \pm 0.62 \mathrm{ps}$ (specification is $196 \mathrm{ps}$ ) and 133.80 $\pm 0.62 \mathrm{ps}$ (specification is $134.82 \mathrm{ps}$ ) respectively.

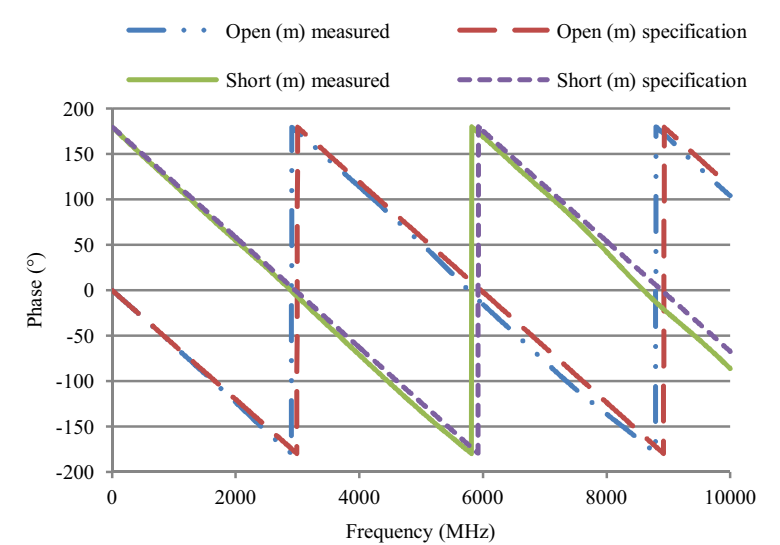

Fig. 4.a. Calculated and specification phase for BNC open (m) and BNC short $(\mathrm{m})$ calibration standards.

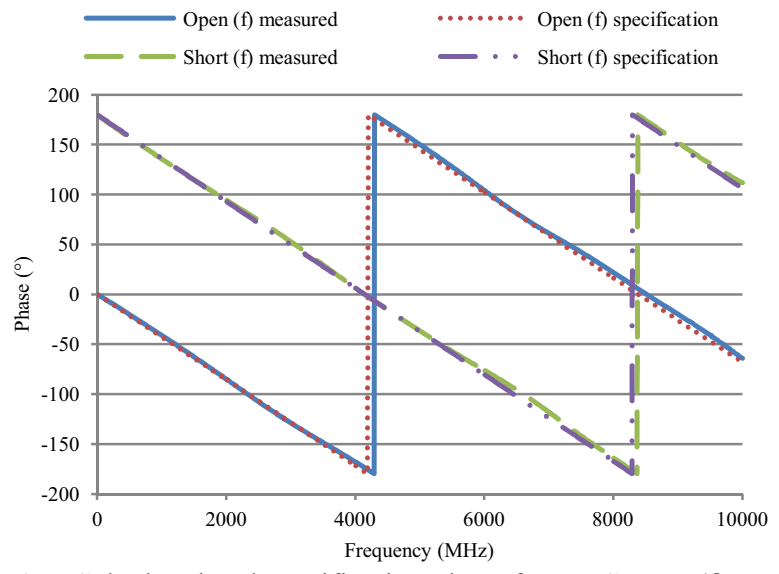

Fig. 4.b. Calculated and specification phase for BNC open (f) and $\mathrm{BNC}$ short (f) calibration standards.

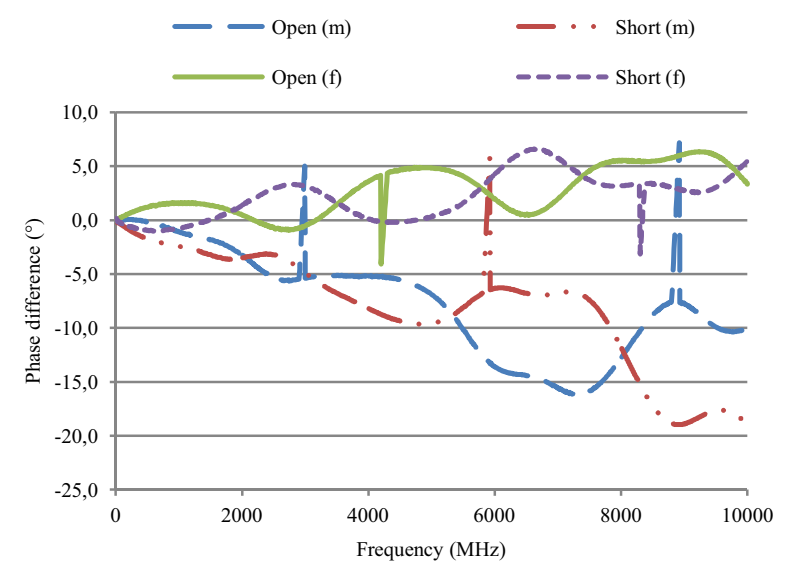

Fig. 5. Phase difference between measured and specification for BNC opens and shorts calibration standards.

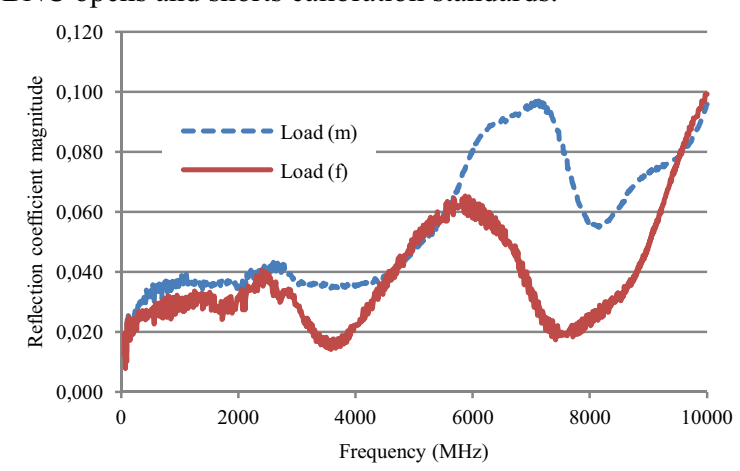

Fig. 6. Calculated reflection coefficient magnitudes of BNC male and BNC female matched loads. 
After adapter measurement, open, short and matched load are alternately connected to suitable adapter and reflection coefficient of adapter side $\left(\Gamma_{M}\right)$ is measured up to its maximum frequency $(18 \mathrm{GHz})$ and then reflection coefficient of calibration standard is calculated.

To check the measurement result, calibration standards are also measured directly using calibrated VNA and it is called as actual result. Difference between measured and actual result for opens and shorts with male and female connectors are given in Figure 7.

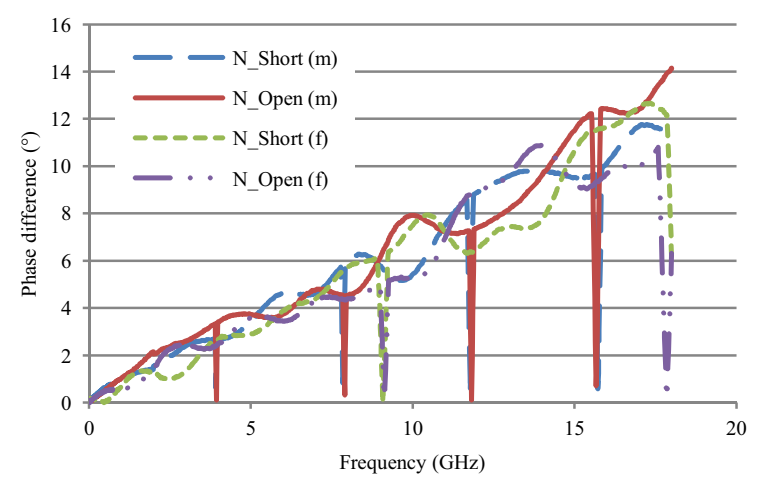

Fig 7. Difference between measured and actual result for type $\mathrm{N}$ standards.

Sharp peak and deep points in Figure 7 are caused by phase change from $-180^{\circ}$ to $+180^{\circ}$. As seen from the Figure 7, maximum difference between measured and actual value is lower than $15^{\circ}$ at $18 \mathrm{GHz}$. This difference (measurement error) is arisen by approximately $2.3 \mathrm{ps}$ delay and it can be added to adapter delay uncertainty. Finally, total uncertainty including measurement error and uncertainty for adapter delay is obtained as $2.92 \mathrm{ps}$ for type $\mathrm{N}$ adapter.

Same procedure as measurement of BNC type matched loads is also applied to type $\mathrm{N}$ matched loads. Measured and actual result for type $\mathrm{N}$ matched load have male and female connectors are given in Figure 8.

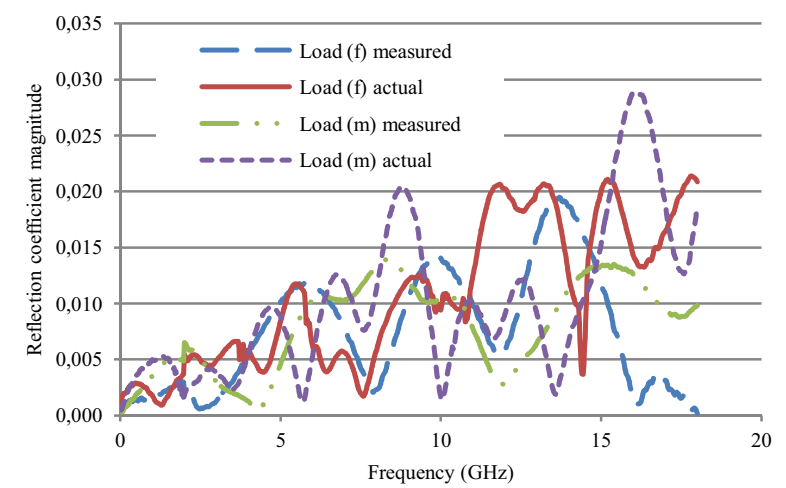

Fig. 8. Type $\mathrm{N}$ matched loads.

\section{Conclusion}

In this study, BNC type calibration standards for VNA are characterized through adapter characterization approach. Type $\mathrm{N}$ to $\mathrm{BNC}$ adapters are used to obtain traceability from the International System of Units (SI). Then BNC calibration standards are measured using traceable type $\mathrm{N}$ to $\mathrm{BNC}$ adapters to transfer traceability from reference standards to $\mathrm{BNC}$ calibration standards. Measured result of $\mathrm{BNC}$ opens and $\mathrm{BNC}$ shorts are compared with manufacturer specifications. Results are agreed in $19^{\circ}$ up to $10 \mathrm{GHz}$. BNC and type $\mathrm{N}$ matched loads are also measured and magnitudes of results are compatible with actual results for type $\mathrm{N}$ connector.

To verify the method, known and traceable type $\mathrm{N}$ calibration standards are measured and compared with actual result. Differences between measured and actual results for opens and shorts are lower than $8^{\circ}$ up to 10 $\mathrm{GHz}$ and it is arisen from 2.3 ps delay. This delay could be added to $\mathrm{BNC}$ adapter uncertainty as a measurement error. As a conclusion, the BNC type calibration standards are characterized for S-parameter measurement and these standards are traceable to SI through characterized adapters. Uncertainty of adapters could be analyzed regarding to the verification measurements and calibration standards uncertainty should be calculated.

\section{References}

1. MIL-PRF-39012E, "Performance specification for radio frequency coaxial connectors", (2005)

2. https://www.pasternack.com/images/ProductPDF/P E7049-20.pdf, (visiting date, $13^{\text {th }}$ July 2017)

3. http://docseurope.electrocomponents.com/webdocs/0ec1/0900 766b80ec1bf6.pdf, (visiting date, $13^{\text {th }}$ July 2017)

4. https://www.maurymw.com/Precision/BNC_Cal_Ki ts.php, (visiting date, 13th July 2017)

5. http://rosenberger.de/ok/images/documents/db/51C K010-150_DB.pdf, (visiting date, $13^{\text {th }}$ July 2017)

6. IEEE 287, "IEEE Standard for precision coaxial connectors (DC to $110 \mathrm{GHz}$ )", (2007) 